\title{
A wide-angle shift-free metamaterial filter design for anti-laser striking application
}

Monks, James; Yue, Liyang; Yan, Bing; Aldred, Barrie ; Hurst, Andrew; Wang, Zengbo

\section{Optics Communications}

DOI:

10.1016/j.optcom.2018.07.065

Published: 15/12/2018

Peer reviewed version

Cyswllt i'r cyhoeddiad / Link to publication

Dyfyniad o'r fersiwn a gyhoeddwyd / Citation for published version (APA):

Monks, J., Yue, L., Yan, B., Aldred, B., Hurst, A., \& Wang, Z. (2018). A wide-angle shift-free metamaterial filter design for anti-laser striking application. Optics Communications, 429, 53-59. https://doi.org/10.1016/j.optcom.2018.07.065

\footnotetext{
Hawliau Cyffredinol / General rights

Copyright and moral rights for the publications made accessible in the public portal are retained by the authors and/or other copyright owners and it is a condition of accessing publications that users recognise and abide by the legal requirements associated with these rights.

- Users may download and print one copy of any publication from the public portal for the purpose of private study or research.

- You may not further distribute the material or use it for any profit-making activity or commercial gain

- You may freely distribute the URL identifying the publication in the public portal ?
}

Take down policy

If you believe that this document breaches copyright please contact us providing details, and we will remove access to the work immediately and investigate your claim. 
Manuscript Number: MQ-2934

Title: A Wide-Angle Shift-Free Metamaterial Filter Design for Anti-Laser Striking Application

Article Type: Research Paper

Section/Category: Nano and Micro optics

Keywords: Optical Metamaterials; Laser Protection, Wide-angle Blocking; Bandstop Filter.

Corresponding Author: Dr. zengbo Wang,

Corresponding Author's Institution: Bangor University

First Author: James N Monks

Order of Authors: James N Monks; Liyang Yue, PhD; Bing Yan, PhD; Barrie Aldred; Andrew Hurst; zengbo Wang

Abstract: Laser striking is a growing safety concern for pilots and aircraft passengers and there is a great need to develop a wide-angle and shift-free laser filter and protection device. This paper presents one of such design that can effectively block out a class 3B 532 nm green laser with an Optical Density (OD) of $1.88+$ for all polarisation states over a wide range of incident angles up to 85 degree. The design consists of anti-reflection layer, active blocking and substrate layers. The central active blocking layer is designed to be a metamaterial composite media made of silver nanoparticles (AgNP) organised in a three-dimensional (3D) primitive hexagonal Bravais lattice configuration and embedded in a highindex dielectric of Zinc Sulphide (ZnS). The designed filter can be placed onto a number of devices ranging from personnel goggles to aircraft windows. The work may lead to the realisation of next generation laser protection devices that are currently sought by major aerospace companies and government defence agencies. 
School of Electronic Engineering,

College of Applied and Physical Sciences,

Bangor University,

Bangor, Gwynedd, LL57 1UT.

Dear Editor of Optics Communications,

I am submitting a manuscript for consideration of publication in the journal titled "Optics Communications". The manuscript is titled "A Wide-Angle Shift-Free Metamaterial Filter Design for Anti-Laser Striking Application".

The manuscript has not been published elsewhere and it has not been submitted simultaneously for publication elsewhere.

Manuscript overview: With regular worldwide news exposure, handheld portable laser hold a considerable threat to pilots and other transportation vehicles. Current thin-film laser protection technology is unable to cope with this new threat due to the technologies disadvantages in angular performance, where the transmission spectrum of the thin film filter undergoes a continuous blueshift as angle of incidence increases. The compensation for greater angular performance in thin film technology results in high colouration and low integrated visual photopic responses. The optical and transport industries have a desired interest in developing a true wide-angle shift-free laser protection filter. Our research puts forward a solution to this problem. We have designed a filter connecting plasmonic metamaterials with traditional thin-film coating theory. The results outlined in our manuscript present a wide-angle shift-free laser protection device for blocking a green $532 \mathrm{~nm}$ laser.

Thank you for your consideration.

Yours Sincerely,

Dr Zengbo Wang and team.

Email: z.wang@bangor.ac.uk 
Potential Reviewers for the manuscript titled:

"A Wide-Angle Shift-Free Metamaterial Filter Design for Anti-Laser Striking Application" J. N. Monks ${ }^{1}$, L. Yue ${ }^{1}$, B. Yan ${ }^{1}$, B. Aldred ${ }^{2}$, A. Hurst ${ }^{2}$ \& Z. Wang ${ }^{1 *}$

Bangor University ${ }^{1}$ \& Qioptiq Ltd. $^{2}$

*Email: z.wang@bangor.ac.uk

Journal submitted: Optics Communications

\begin{tabular}{|l|l|l|l|}
\hline \multicolumn{1}{|c|}{ Title } & \multicolumn{1}{|c|}{ Name } & \multicolumn{1}{|c|}{ Institute } & \multicolumn{1}{c|}{ Email } \\
\hline Professor & Boris Luk'yanchuk & $\begin{array}{l}\text { Physical Faculty, Lomonosov } \\
\text { Moscow State University }\end{array}$ & Boris_L@dsi.a-star.edu.sg \\
\hline Professor & Igor Minin & $\begin{array}{l}\text { National Research Tomsk } \\
\text { Polytechnic University }\end{array}$ & prof.minin@gmail.com \\
\hline Dr & Nikita Arnold & $\begin{array}{l}\text { Institute of Applied Physics, } \\
\text { Johannes Kepler Universitat Linz }\end{array}$ & nikita.arnold@jku.at \\
\hline Dr & Herman Chen Zaichun & $\begin{array}{l}\text { Singapore University of Technology } \\
\text { and Design }\end{array}$ & herman_chen@ sutd.edu.sg \\
\hline Dr & Nikitas Papasimakis & University of Southampton & np3@orc.soton.ac.uk \\
\hline
\end{tabular}




\title{
A Wide-Angle Shift-Free Metamaterial Filter Design for Anti-Laser Striking Application
}

\author{
James N Monks ${ }^{1}$, Liyang Yue ${ }^{1}$, Bing Yan ${ }^{1}$, Barrie Aldred ${ }^{2}$, Andrew Hurst $^{2}$ \& Zengbo Wang ${ }^{1}$ \\ ${ }^{1}$ School of Electronic Engineering, Bangor University, Dean Street, Bangor, Gwynedd, UK, LL57 1UT \\ ${ }^{2}$ Qioptiq Ltd, St Asaph, Denbighshire, UK, LL17 OLL \\ E-mail: z.wang@bangor.ac.uk
}

Laser striking is a growing safety concern for pilots and aircraft passengers and there is a great need to develop a wide-angle and shift-free laser filter and protection device. This paper presents one of such design that can effectively block out a Class 3B $532 \mathrm{~nm}$ green laser with an Optical Density (OD) of $\mathbf{1 . 8 8 +}$ for all polarisation states over a wide range of incident angles up to 85 degree. The design consists of anti-reflection layer, active blocking and substrate layers. The central active blocking layer is designed to be a metamaterial composite media made of silver nanoparticles (AgNP) organised in a three-dimensional (3D) primitive hexagonal Bravais lattice configuration and embedded in a high-index dielectric of Zinc Sulphide (ZnS). The designed filter can be placed onto a number of devices ranging from personnel goggles to aircraft windows. The work may lead to the realisation of next generation laser protection devices that are currently sought by major aerospace companies and government defence agencies.

Keywords: Optical metamaterials; Laser protection; Wide-angle blocking, Bandstop filter

\section{Introduction}

Over the past decade, visible lasers have become a serious risk to aircraft and other transportation vehicles due to the ease of accessibility and low cost of ownership. Recent reports from the Federal Aviation Administration (FAA) and their British counter part, Civil Aviation Authority (CAA), have illustrated that visible laser attacks from green $(532 \mathrm{~nm}$ ) lasers make up $83-91 \%$ of all reported incidents [1,2], with some of the most powerful laser attacks involving diode-pumped solid-state (DPSS) lasers [3]. The resulting damage caused from these attacks are mainly associated with laser eye dazzle [4,5], which describes the temporary impairment to the human eye caused from lasing light within the visible wavelengths. However, exposure for even a short time at the visible radiation range can produce retinal and photo-chemical damage to the eye, and even cause thermal damage to skin [6]. An increased risk of these laser occurrences is that the lasing light could be incident from any angle. Traditional thin-film optics (Rugate notch filters) are currently used to protect against lasers [7-9], and comprise of interference band filters organised as multiple thin coating layers of high and low refractive index dielectric films on a desired substrate [10]. This technology can provide high optical density (typically 4OD+ to $80 \mathrm{D}+$ ) and allows for the blocking wavelength to be tailored according to the requirements [11]. However, Rugate filters have disadvantages especially with angular intolerance. As the angle of incidence increases, the transmission spectrum of the filter experiences a continuous blue-shifting to shorter wavelength [12], causing the failure of the device to operate at desired blocking wavelength. To circumvent this issue, the existing solution in Rugate filter design is to increase the bandwidth of the stop band. This in turn leads to problems including reduced visibility, high colouration and low integrated visual photopic transmission [13]. Companies like Qioptiq, BAE Systems, Boeing, Airbus and government defence agencies have a shared interest in developing a truly wide-angle (up to 60 degree) with shift-free laser blocking and protection device which drives this research. Our aim is to employ metamaterial (MM) design concept to solve the challenge and develop the product. 
Metamaterials (MMs) are man-made materials that are not found in nature, and are able to gain electromagnetic (EM) characteristics due to the structural arrangement of meta-atoms, or unit cells, organised in a repeating pattern, where the meta-atoms are much smaller than the wavelength of interest $[14,15]$. Band rejection filters designed from MMs are competently able to block EM radiation at certain frequency bands. The earliest works in this area were conducted within the lowfrequency bands and consisted of the well-known split ring resonator (SRR) $[16,17]$, following with the emergence of various modifications to this design [18-21]. A simplified design that is now commonly used for band rejection in MM is the 'fishnet' design [15], and was first studied, in 2008 by Valentine et al., to effectively simplify and streamline the shape of the meta-atom and therefore, reduce the difficulties associated with fabrication [22]. The development of these structures has been greatly researched in the Gigahertz $(\mathrm{GHz})$ and Terahertz $(\mathrm{THz})$ bands [23-26], and has moved towards optical frequency. Manufacturing of large-area optical metamaterials, however, has been and continued as a challenge task. Consequently, measures are taken to simplify the structure, with such arrangements existing as circular disk meta-atoms and spherical particles, where these designs can provide similar performance to the fishnet design [27-29]. Bandstop filters in the near-optical region have been achieved using structured coupled cut-wire designs [30], however, this design does not put forward any claim to wide-angle operation, nor polarisation insensitivity. A wide-angle study within the infrared band was reported in 2010 by Liu et al [31]. Though, this article demonstrated a shift-free perfect plasmonic absorption with zero transmission across the broadband spectrum. The movement to the optical band provides difficulty for wide-angle performance because the MM device requires a sustained resonance at high-frequencies for all angles of incidences. The movement towards 3D metamaterials may provide the solution which has expanded in recent year.. One of the earliest examples was demonstrated using a multilayers metal-dielectric fishnet metamaterial [32]. Such devices enabled for a stronger magnetic activity and varying backward phase propagation. Additionally, a much lower transmission rate was achieved with 3D metamaterial filter [32,33]. The research outlined in this paper puts forward a 3D metamaterial design for polarisation-insensitive wide-angle metamaterial band rejection filter to block out a class 3B 532nm wavelength green light laser based on plasmonic nanoparticles. In the following, the filter parameters were firstly introduced. The metamaterial design was then presented and theoretical formulations for design parameter determinations were given, followed by the simulated device performances, discussions on manufacturing issues and final conclusions.

\section{Filter design parameters}

The desired anti-laser filter will be measured by angular performance of key parameters including transmission spectrum $T(\lambda)$, Optical Density (OD), Integrated Visual Photopic Transmission (IVPT) and Colouration, under different polarisation states. These goal parameters are defined as follows.

\section{(a) Transmission Spectrum $T(\lambda)$ and Optical Density (OD)}

An ideal transmission response of the blocking device is illustrated in figure 1, with an insert demonstrating an artistic impression of an anti-laser filter and its optical filtering capability to block out a $532 \mathrm{~nm}$ wavelength green laser whilst passing through all other wavelengths, whilst attaining an overall high transparency. The Blocking performance of the filter can be measured from the optical density (OD), and is defined as:

$$
O D=-\log _{10}(T(\lambda))
$$

The optical density is defined as the degree to which a medium impedes transmitted light waves, and is commonly used within the optical industry to define laser safety guidelines. Our design filter 
will block out a central wavelength of $532 \mathrm{~nm}(527 \mathrm{~nm}-534 \mathrm{~nm})$, with a bandwidth smaller than $50 \mathrm{~nm}$.

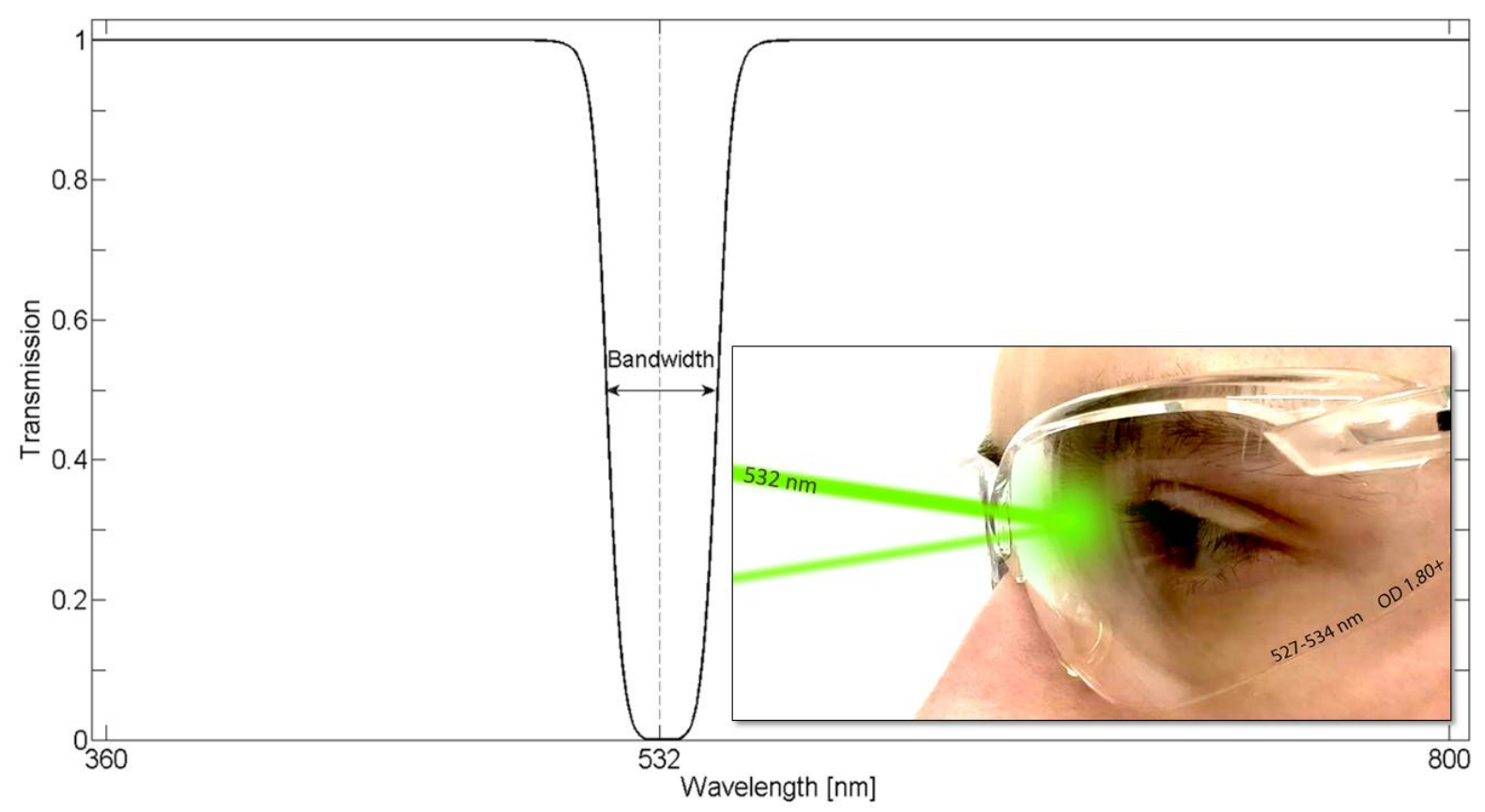

Fig.1. The conceptual aim. The figure indicates the ideal transmission response with the insert showing an artist impression of the optical metamaterial notch filter on personnel goggles, deflecting a $532 \mathrm{~nm}$ laser beam ( $527 \mathrm{~nm}-534 \mathrm{~nm})$, with a bandwidth smaller than $50 \mathrm{~nm}$.

\section{(b) Integrated Visual Photopic Transmission (IVPT)}

The IVPT is the function associated with the average spectral sensitivity of the filter in accordance to the typical human visual perception, given by (Eq.2). The importance of this is to understand how the human eye will interact visually with the filter.

$$
I V P T=\frac{\int_{0}^{\infty} I(\lambda) V(\lambda) T(\lambda) d \lambda}{\int_{0}^{\infty} I(\lambda) V(\lambda) d \lambda}
$$

Where $I(\lambda)$ is the spectrum illumination CIE-D65 (daylight), $V(\lambda)$ is the photopic response of the human eye and $T(\lambda)$ is the transmission spectrum of the filter $(0 \leq T \leq 1)$.

\section{(c) Optical Colouration}

All optical notch filters will experience a degree of functional visual impairment. The visual impact of an optical filter is a relation to the loss of transmission (IVPT) and the colouration. For analysis of the anti-laser filter, the standard colour map (CIE 1931 Standard Observer) will be used. Due to individual variation of the human eye, the distribution of cones in the eye result in the tristimulus values being dependant on the observer's field of view. The CIE 1931 colour map represents a chromatic response for the average human within a $2^{\circ}$ arc inside the fovea centralis, a region of closely packed cones in the eye; thus, providing the user confidence of the colouration response. The transmission and reflective cases can be established from Eq.3 and Eq.4.

$$
\begin{aligned}
& X=\int_{0}^{\infty} I(\lambda) T(\lambda) \bar{x}(\lambda) d \lambda \\
& Y=\int_{0}^{\infty} I(\lambda) T(\lambda) \bar{y}(\lambda) d \lambda
\end{aligned}
$$




$$
Z=\int_{0}^{\infty} I(\lambda) T(\lambda) \bar{z}(\lambda) d \lambda
$$

Where $I(\lambda)$ and $T(\lambda)$ is same as those in above (Eq.2), and $\bar{x}(\lambda), \bar{y}(\lambda)$ and $\bar{z}(\lambda)$ are colour matching functions that numerically describe the chromatic response of the observer.

The translation to the $x-y$ coordinates on the colour map are described by Eq.4.

$$
\begin{aligned}
& x=\frac{X}{X+Y Y+Z} \\
& y=\frac{Y}{X+Y+Z}
\end{aligned}
$$

\section{Results and Discussions}

\section{Proposed design}

We have use plasmonics and AR coating theory to develop the filter design. Figure 2 shows the proposed anti-laser notch filter design that shows wide-angle and polarisation insensitive properties at $532 \mathrm{~nm}$ wavelength. The design consists of three layers: anti-reflection (AR, top), active blocking ( $A B$, middle) and substrate layers (bottom). The active layer in the middle is a $3 D$ artificial media made of silver nanoparticles (AgNP) organised in a primitive hexagonal Bravais lattice planar array form and embedded in a surrounding dielectrics. We select AgNP as main active element because of its flexibility in its resonance engineering: its peak position, bandwidth and transmission rate can be well tuned by size, surrounding medium and 3D configuration. The hexagonal 3D arrangement was chosen as it boosts device's angular performance. The AgNP size was fixed at $9 \mathrm{~nm}$ (diameter) in the design. This follows the design requirement of narrow blocking bandwidth of less than $50 \mathrm{~nm}$ (Fig.1). However, at $9 \mathrm{~nm}$ diameter the AgNP dielectric constant deviates from it bulk and needs to be corrected. This was achieved by including a size correction factor in the design process. Finally, the AR layer is deposited on the top of the active blocking layer to ensure beams outside of the blocking band (e.g daylight) are able to pass through the device at a high transmission rate. This is important to ensure that device has a clear visibility while blocking out the $532 \mathrm{~nm}$ laser beam. 


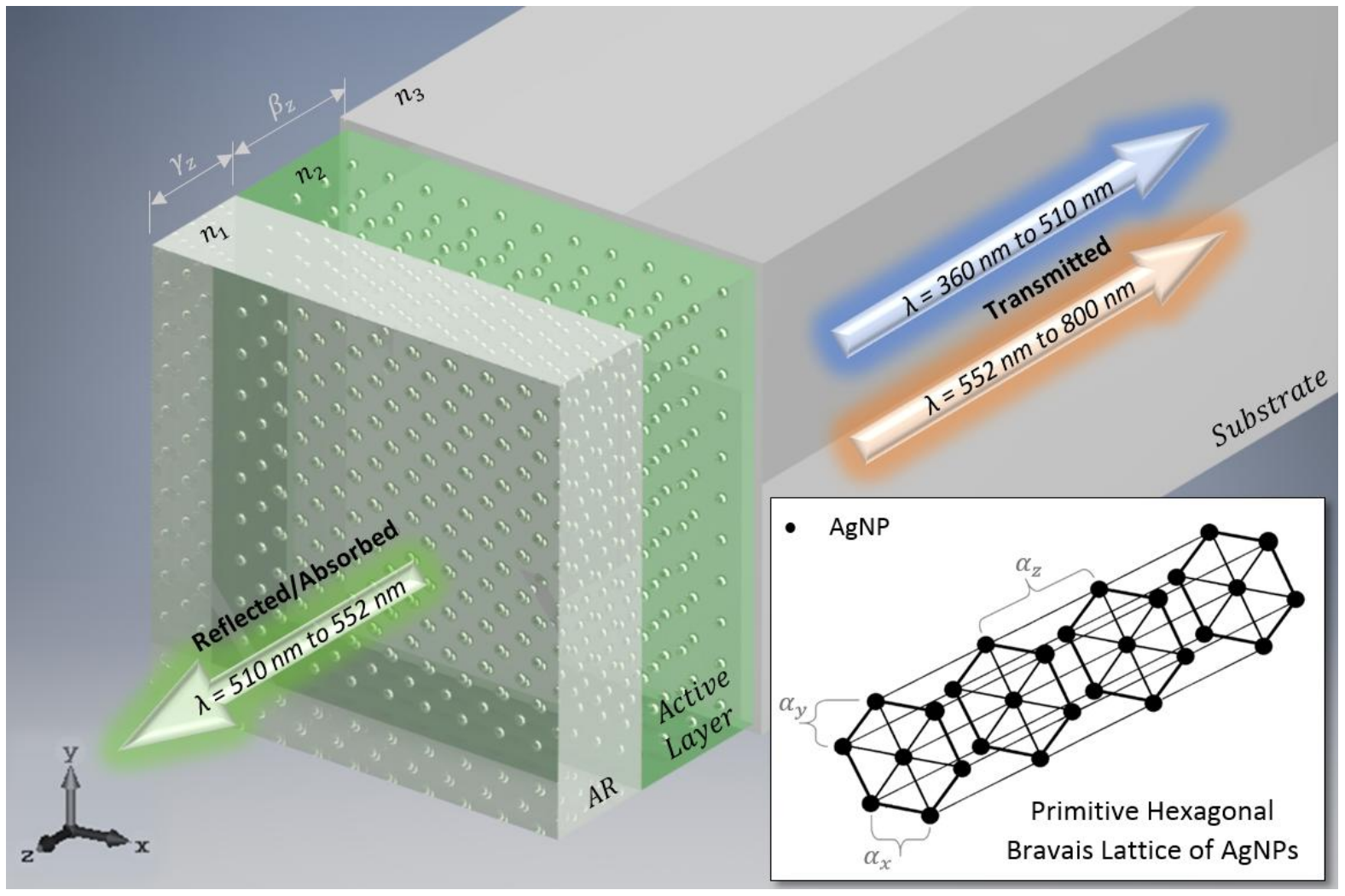

Fig. 2. (a) Configuration of the $532 \mathrm{~nm}$ anti-laser device based on three-dimensional hexagonal planar array of AgNP as the active layer with $\alpha_{x}=\alpha_{y}=\alpha_{z}=\lambda_{\text {laser }} / 16 ; \beta_{z}=4 \alpha_{z}$ and $\gamma_{z}=3 \alpha_{z}$. Insert shows the hexagonal lattice arrangement of the AgNPs within the 'active layer'.

\section{Design theory and process}

Our basic design theory is plasmonics, namely plasmon resonance of silver nanoparticles (AgNP). A coupled design approach involving both analytical theory and numerical simulation was used. We use analytical theories (e.g Mie theory and thin-film interference theory) as base to find approximate design parameters and subsequently use full-wave numerical simulation software CST Microwave Studio to tune and verify them to meet the design targets. The optical properties for refractive indices and extinction coefficients have been obtained from literature [39-41] and patched for small AgNPs as discussed above and in [42]. The light propagation direction, $k$ vector, with normal incident is perpendicular to the design facets. The electric field, $E$, is directional to the $y$-axis and the magnetic field, $H$, is directional to the $x$-axis, and initiated for the required transverse modes. The wave propagation angle, theta, has been adjusted accordingly to compute the $\mathrm{k}$ vector at various angle of incidence. A generalised design approach is finally developed, which can be used to find filter parameters for any blocking wavelength within the visible band. We use $532 \mathrm{~nm}$ as a particular example in this paper, but other wavelengths such as $450 \mathrm{~nm}$ and $613 \mathrm{~nm}$ are easily achievable as well.

Determination of host medium for AgNP: The active layer consisting of a 3D primitive hexagonal Bravais lattice of 9-nm-diameter AgNP. The composite's effective refractive index can be determined from the Maxwell-Garnett formula (Eq.5), which defines the bulk effective permittivity of the metaldielectric composite [34], and relationship between refractive index and permittivity (Eq.6)

$$
\begin{gathered}
\frac{\varepsilon_{e f f}-\varepsilon_{h}}{\varepsilon_{e f f}+2 \varepsilon_{h}}=f \frac{\varepsilon_{m}-\varepsilon_{h}}{\varepsilon_{m}+2 \varepsilon_{h}} \\
\tilde{n}^{2}=(n+i k)^{2} \equiv \varepsilon_{e f f}=\varepsilon_{r}+i \varepsilon_{i}
\end{gathered}
$$


$\varepsilon_{\text {eff }}$ represents the effective permittivity with its respective complex notation, $\varepsilon_{m}$ and $\varepsilon_{h}$ signifies the permittivity for $\mathrm{Ag}$ and the dielectric host respectively, and $n$ and $k$ denote the refractive index and extinction coefficient. An additional term has been added to the classical Drude dampening frequency, $\gamma_{\text {particle }}=\gamma+\left(v_{F} /\left(\alpha r_{p}\right)\right)$ to compensate for the particle becoming smaller than the free mean path of the electron (for silver the bulk mean free path at room temperature is about 53 $\mathrm{nm}$ [35]); $\gamma$ and $\gamma_{\text {particle }}$ represents the dampening frequency of the bulk metal and new dampening frequency according to the particle size $\left(r_{p}\right)$, with relation to the Fermi velocity $\left(v_{F}\right)$ and $\alpha$, where due to the spherical nature of the particles $\alpha=4 / 3$. Accordingly, the particle size has been taken into consideration for this design.

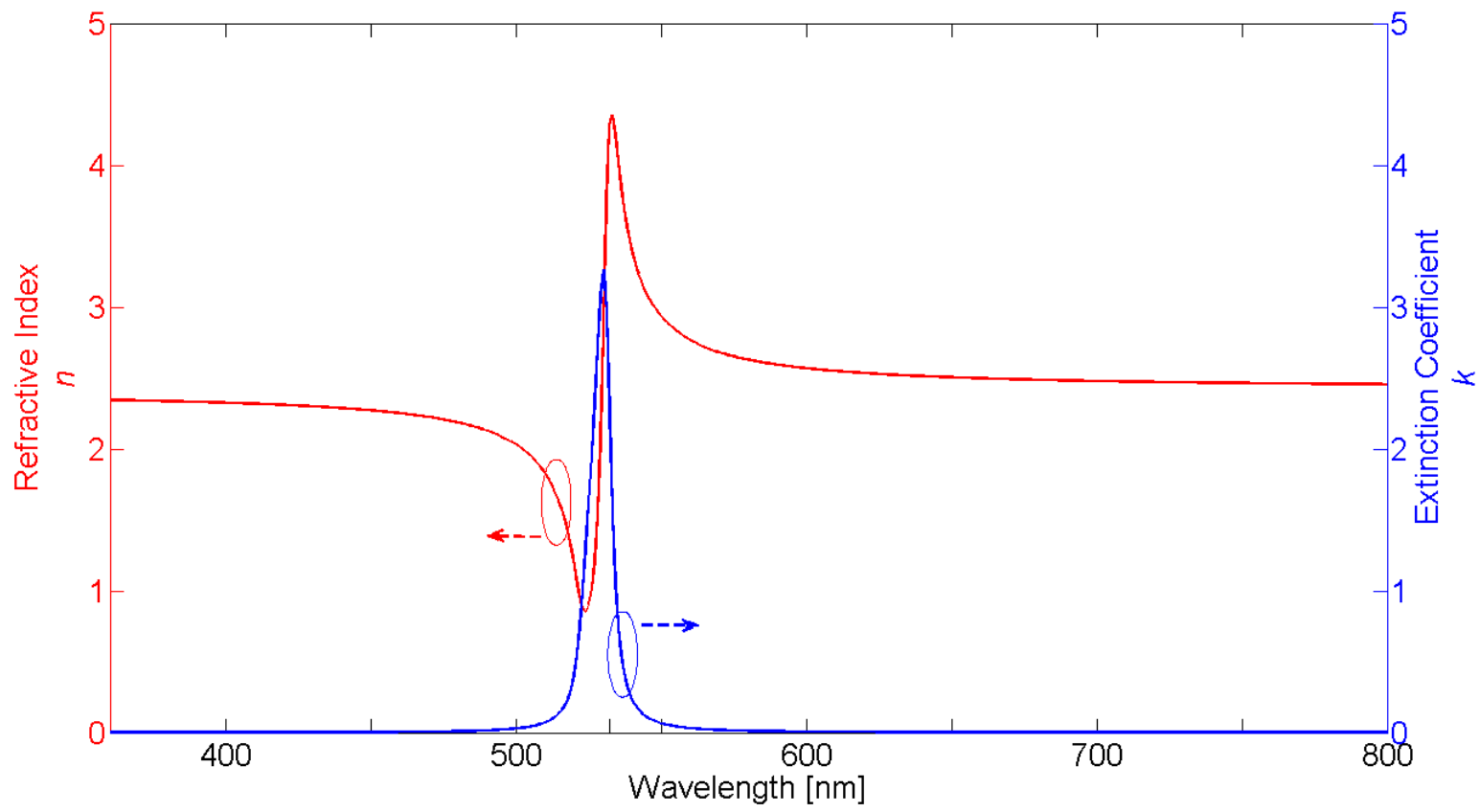

Fig.3. The effective refractive index and extinction coefficient for the active layer.

Once the effective refractive index of active layer was determined, we use following equation to find the notch location, according to the plasmonic material and surrounding medium;

$$
\lambda_{t}=e^{\frac{c_{1}+R e\left[n_{\text {host }}\right]}{c_{2}}}-\sigma
$$

Where $\lambda_{t}$ is the desired wavelength and is equal to the wavelength associated to the peak value of the extinction coefficient $(k)$ of the active layer. $\lambda_{t}=532 \mathrm{~nm}$ when $\operatorname{Re}\left[n_{\text {host }}\right]=2.382$ and $\sigma=$. The $c$ values are the coefficients of Silver $\left(c_{1}=18.741\right.$ and $\left.c_{2}=3.3654\right), n_{\text {host }}$ is the refractive index of the surrounding dielectric medium and $\sigma$ is a particle size correction function $(\sigma=1.24)$. This formula applies to all other wavelengths. For example, if the desired wavelength was to block out a $450 \mathrm{~nm}$ blue laser, the surrounding dielectric medium would need a refractive index of $n=1.82$ instead.

Determine particle spacing: The blocking bandwidth $(\Delta \lambda)$ is affected by both the AgNP particle size and its 3D hexagonal lattice array density. The $Q$-factor, defined as $Q=\lambda_{t} / \Delta \lambda$, was used to calculate for the blocking wavelength bandwidth. The Q-factor was optimised for high blocking performance while remaining with a minimal bandwidth, resulting in an optimum design of $\alpha_{x}=\alpha_{y}=\alpha_{z}=\lambda_{\text {laser }} / 16 ; \beta_{z}=4 \alpha_{z}$ and $\gamma_{z}=3 \alpha_{z}$, as shown in figure 2 . 
Determine AR coating and layer thicknesses: The $532 \mathrm{~nm}$ anti-laser device has an active layer that is sandwiched between a glass substrate $(n=1.52)$ and an AR coating layer of Magnesium fluoride $\left(\mathrm{MgF}_{2}, \mathrm{n}=1.38\right)$. The introduction of $\mathrm{MgF}_{2}$ is to reduce the reflected light intensity and improve the overall transmittance of the device by allowing the natural incident incoherent light to experience a phase difference of $\pi$, resulting in destructive interference of the waves. The thin film anti-reflection coating is chosen to match the relationship of the reflection coefficient between air, the active layer and the glass substrate [36], so that:

$$
n_{A R} \approx \frac{1}{\sqrt{n_{\text {air }} / n_{\text {sub }}}}
$$

The presence of the high-index ZnS positioned next to the low-index substrate, makes it appear that the substrate has a high index, thus, the subsequent layer of $\mathrm{MgF}_{2}$ becomes more effective as an $\mathrm{AR}$ coating [37]. The thickness of the two layer coating design has been formulated from conventional double-layer coating principles and is expressed in Eq.9.

$$
\begin{aligned}
\tan ^{2} \delta_{1} & =\frac{\left(n_{s u b}-n_{a i r}\right)\left(n_{Z n S}^{2}-n_{a i r} n_{s u b}\right) n_{M g F_{2}}^{2}}{\left(n_{M g F_{2}}^{2} n_{s u b}-n_{a i r} n_{2}^{2}\right)\left(n_{a i r} n_{s u b}-n_{M g F_{2}}^{2}\right)} \\
\tan ^{2} \delta_{2} & =\frac{\left(n_{s u b}-n_{a i r}\right)\left(n_{a i r} n_{s u b}-n_{M g F_{2}}^{2}\right) n_{Z n S}^{2}}{\left(n_{M g F_{2}}^{2} n_{s u b}-n_{a i r} n_{Z n S}^{2}\right)\left(n_{Z n S}^{2}-n_{a i r} n_{s u b}\right)}
\end{aligned}
$$

Where $\delta$ is a positive real number that satisfies the quarter wavelength relationship and $n$ is the refractive index of the subscripted materials. Two solutions for each $\delta$ value will be produced that must be correctly paired, with the $\delta_{1}$ component being a relation to the AR coating and the $\delta_{2}$ component being a relation to the active layer. However, for this study, the solutions found in Eq.10a have been selected to minimise the number of odd multiples of quarter wavelengths and to sufficiently host the required number of AgNP structured lattice layers. Each $\delta$ solution is multiplied by a reference wavelength, $\lambda_{R e f}=275 \mathrm{~nm}$, to solve for the layer thickness, such that the active layer is approximately equal to $\lambda_{\text {laser }} / 4$.

and

$$
\delta_{1} / 2 \pi=0.1749 \quad \delta_{2} / 2 \pi=0.4803
$$

$$
\delta_{1} / 2 \pi=0.3251 \quad \delta_{2} / 2 \pi=0.0197
$$

In CST simulation, the excitation port of the incident beam was set in the far-field zone of the device, which lies beyond the Rayleigh length $Z_{0}$ given by:

$$
Z_{0} \geq \frac{\pi W_{0}^{2}}{\lambda}
$$

Where $W_{0}$ is the waist radius of the embedded fundamental Gaussian beam of a frequency-doubled $\mathrm{YVO}_{4} 532 \mathrm{~nm}$ laser.

\section{Simulated filter performance}

Figure 4 shows the key result of the designed filter; the transmission response at various incident angles of $0^{\circ}$ (solid line), $30^{\circ}$ (dashed line) and $60^{\circ}$ (dotted line). The dielectric host material, $\mathrm{ZnS}$, tunes AgNP resonance peak from $354 \mathrm{~nm}$ (same lattice configuration but with air as host medium) to $530.35 \mathrm{~nm}$. The resulting bandwidth is $41.8 \mathrm{~nm}$ at the full width at half maximum, qualifying the performance of the filter to introduce a band rejection at the $532 \mathrm{~nm}$ lasing wavelength, whilst providing high transmission outside of the rejected band. As the incident angle increases, it is clear to see that zero-shift occurs at the rejection band. The blocking at the rejection band continues to 
$85^{\circ}$ of incidence. In reality, the user would only experience the lasing wavelength at varying incident angles. The natural light transmission outside of the $532 \mathrm{~nm}$ wavelength would remain at maximum at the angle of the users mid-to-near peripheral vision $\left(\theta=30^{\circ}-60^{\circ}\right)$, noted in figure 5 , whilst the ocular focus would remain at the macular region of the peripheral vision $\left(\theta=0^{\circ}-18^{\circ}\right)$. From the wavelengths, outside of the notch bandwidth, of $360 \mathrm{~nm}$ to $510 \mathrm{~nm}$ and $552 \mathrm{~nm}$ to $800 \mathrm{~nm}$, the average transmission across the filter is $81.09 \%$ and $89.06 \%$, respectively; with an overall spectral response of $77.87 \%$ from $360 \mathrm{~nm}$ to $800 \mathrm{~nm}$.

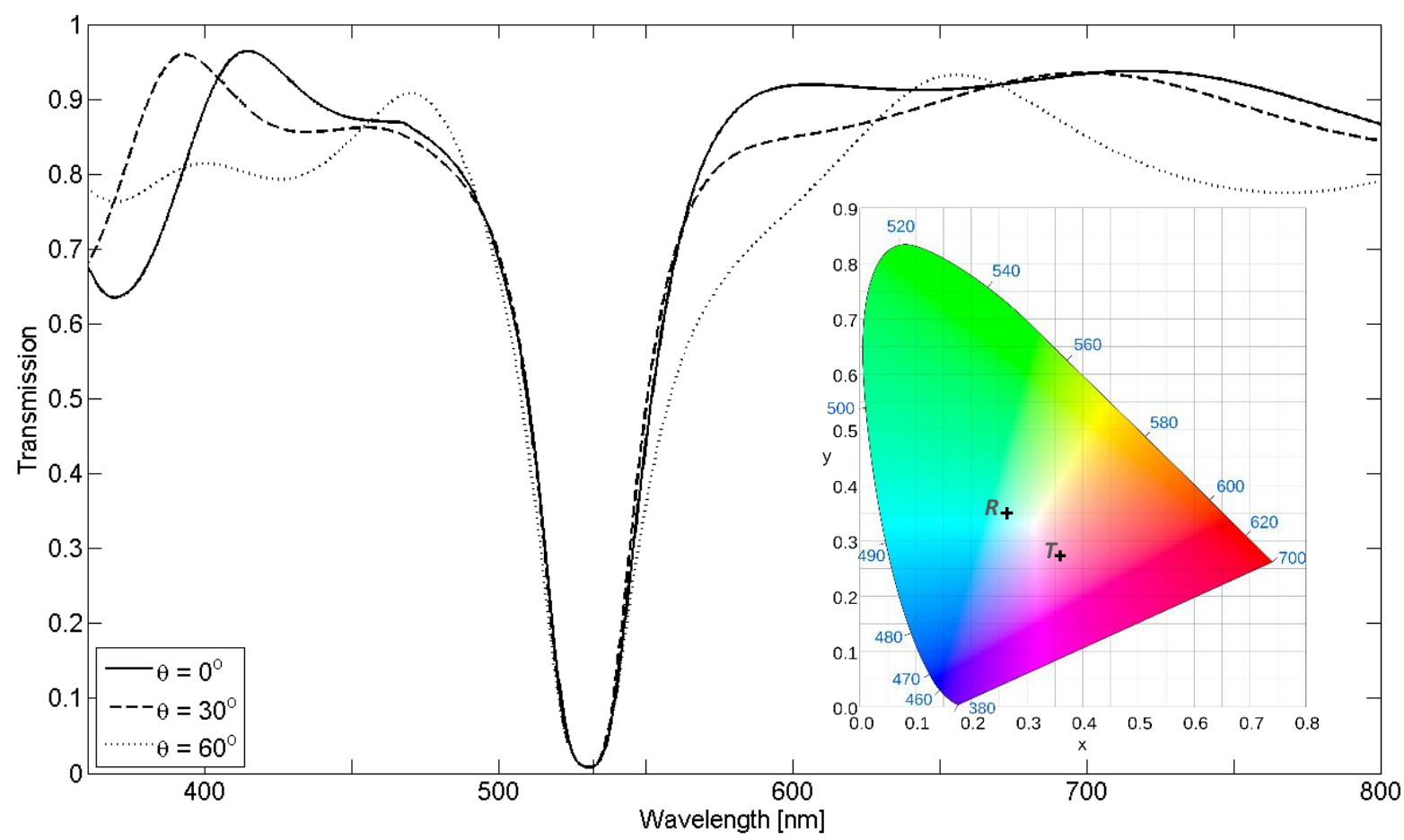

Fig. 4. Transmission of the three-dimensional optical metamaterial filter versus wavelength for incident angle at $\theta=0^{\circ}, \theta=$ $30^{\circ}$ and $\theta=60^{\circ}$. The solid, dashed and dotted lines denote the cases of the incident angle. Insert shows the CIE 1931 colour map for the transmission $(T)$ and reflection (R).

The IVPT response of the discussed filter is $61 \%$. The transmission colouration response exists on the coordinates $(x=0.36$ and $y=0.27)$ and the reflection colouration response exists on the coordinates ( $x=0.26$ and $y=0.35$ ) of the standard CIE 1931 colour map. As seen in figure 4 insert, the calculated transmission and reflection colouration are close to neutral but experience a slight magenta and turquoise hue, respectively.

Figure 5 shows the calculated optical density (OD) of the filter for angles between 0 and 85 degree. The OD remains above 1.88 across the whole range of angles. This indicates that the described filter is able to perfectly block a common low power $(<76 \mathrm{~mW})$ Class 3B $532 \mathrm{~nm}$ DPSS laser of continuouswave operation at all angle of incidence without blue shift occurring. Blocking of $<100 \mathrm{~mW}$ is achievable between the angles of $0^{\circ}$ and $20^{\circ}$. However, despite having zero shift of the notch at the point of resonance, the out of resonance wavelengths begin to attenuate and results in broader bandwidths. This attenuation is greatly augmented beyond the incident angle of $70^{\circ}$ and lies within the far peripheral of the human eye. Due to this attenuation, the device design can be labelled as having a critical angle of $70^{\circ}$.

Another factor to consider is the polarisation sensitivity of the optical filter, figure 5 illustrates that the results are independent of the incident angle in two polarisation modes $p$ and $s$. The slight fluctuation between $p$ and $s$ polarisation results for angles between $45^{\circ}$ to $70^{\circ}$ is a consequence of 
the Brewster angle of the AR coating, $\sim 54^{\circ}$ [43], and is not caused by the active blocking layer of three dimensional AgNP planar array. The variation beyond theta $70^{\circ}$ is due to a minor change $(\sim \pm 1$ $\mathrm{nm}$ ) in resonance between $\mathrm{s}$ and $\mathrm{p}$ polarisation.

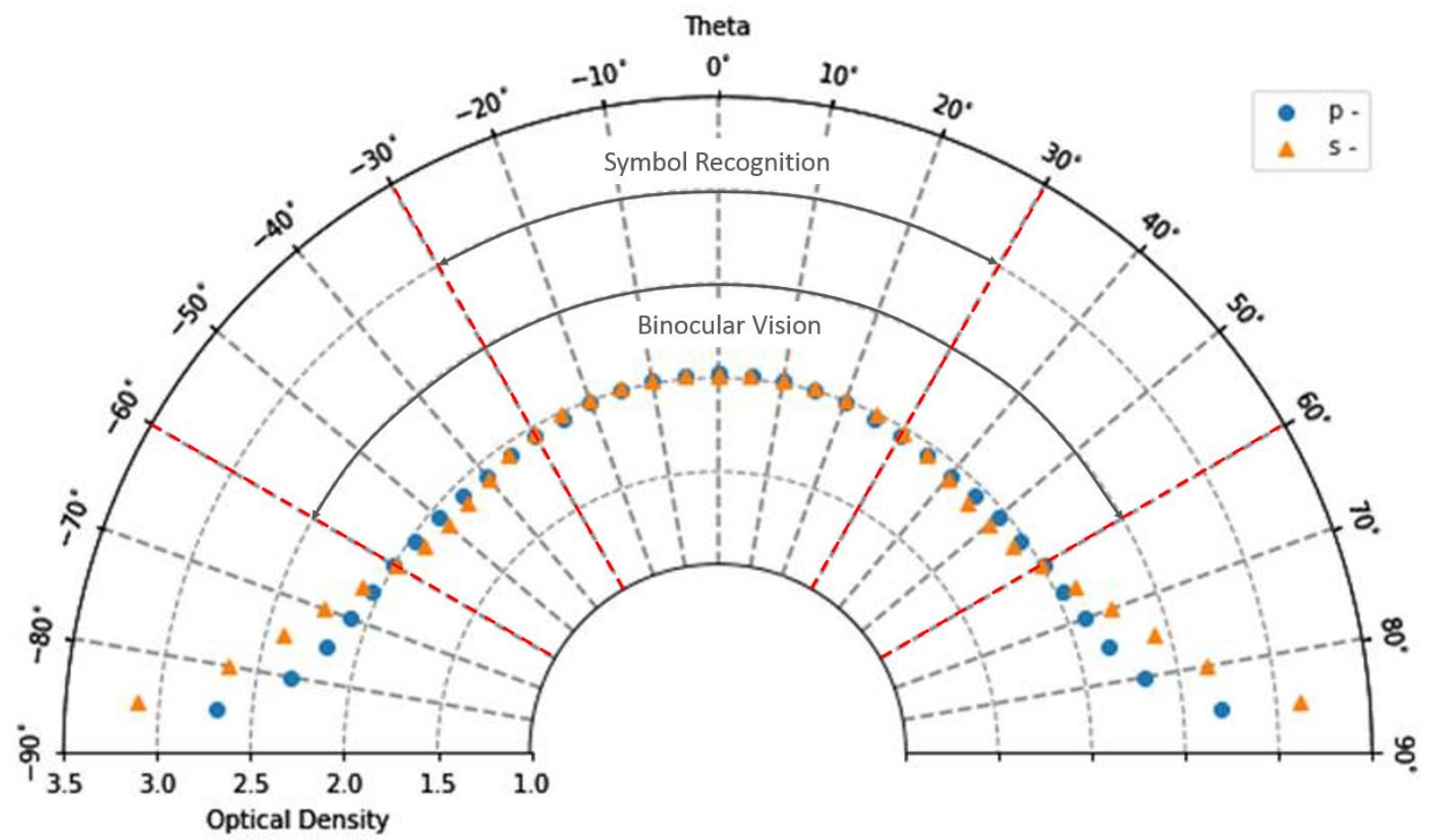

Fig. 5. OD of the designed filter at the laser wavelength of $532 \mathrm{~nm}$. The circle and triangle data points denote the transverse mode of the electromagnetic radiation. Theta represents the angle of incidence. The peripheral vision regions have been noted in accordance to theta.

\section{Mechanism}

The wide-angle shift-free features of the design are resulting from its 3D topological arrangement (primitive hexagonal Bravais lattice) [44] and the excitation of similar resonance modes under different incident angle beams at $532 \mathrm{~nm}$. Figure 6 shows the power flows of Poynting vector calculated for the 9-nm-diameter AgNP when it is irradiated by $532 \mathrm{~nm}$ laser at 0 and 45 degrees for both TE $(a, b)$ and TM $(c, d)$ polarisation incident beams. Under TE wave, the AR layer has a matched phase with Air layer so that angles were preserved at this Air-AR interface. However, the power flows are undergoing a 45-degree rotation to 0-degree when it reaches the $A R-A B$ interface, as shown in (b). As a result, regardless of the angle differences, the power flows passing through the $A B$ layer in (a) and (b) are almost the same, leading to the near-identical field absorption and flow patterns under 0 and 45-degree incidences in the $A B$ layer. This picture holds true for other angles up to about 85 degree and Laser energy was most completely absorbed by three layers of AgNPs in $A B$ media for TE polarisation. This is why multiplayer design has been selected, thus a 3D configuration is important for our work. Under TM wave, however, the situation is slightly different. As shown in (d), the beam rotates by around 45-degree clockwise to 0-degree at air-AR interface, but rotates anti-clockwise back to 45-degree at $A R-A B$ interface, which leads to differences in light absorption between normal (c) and angular incident beams (d), but does not affect the resonance wavelength. Three layers of AgNPs are needed for normal incident beam (c), and 4 layers for $45-$ degree incident beams (d), to reach desired energy decay level. This suggests TM angular beam is the case we need to look at when decides how many AgNPs layers we need in our design. Nevertheless, regardless of polarisation states, the incident laser energy can be blocked by using 4 layer AgNPs design. 


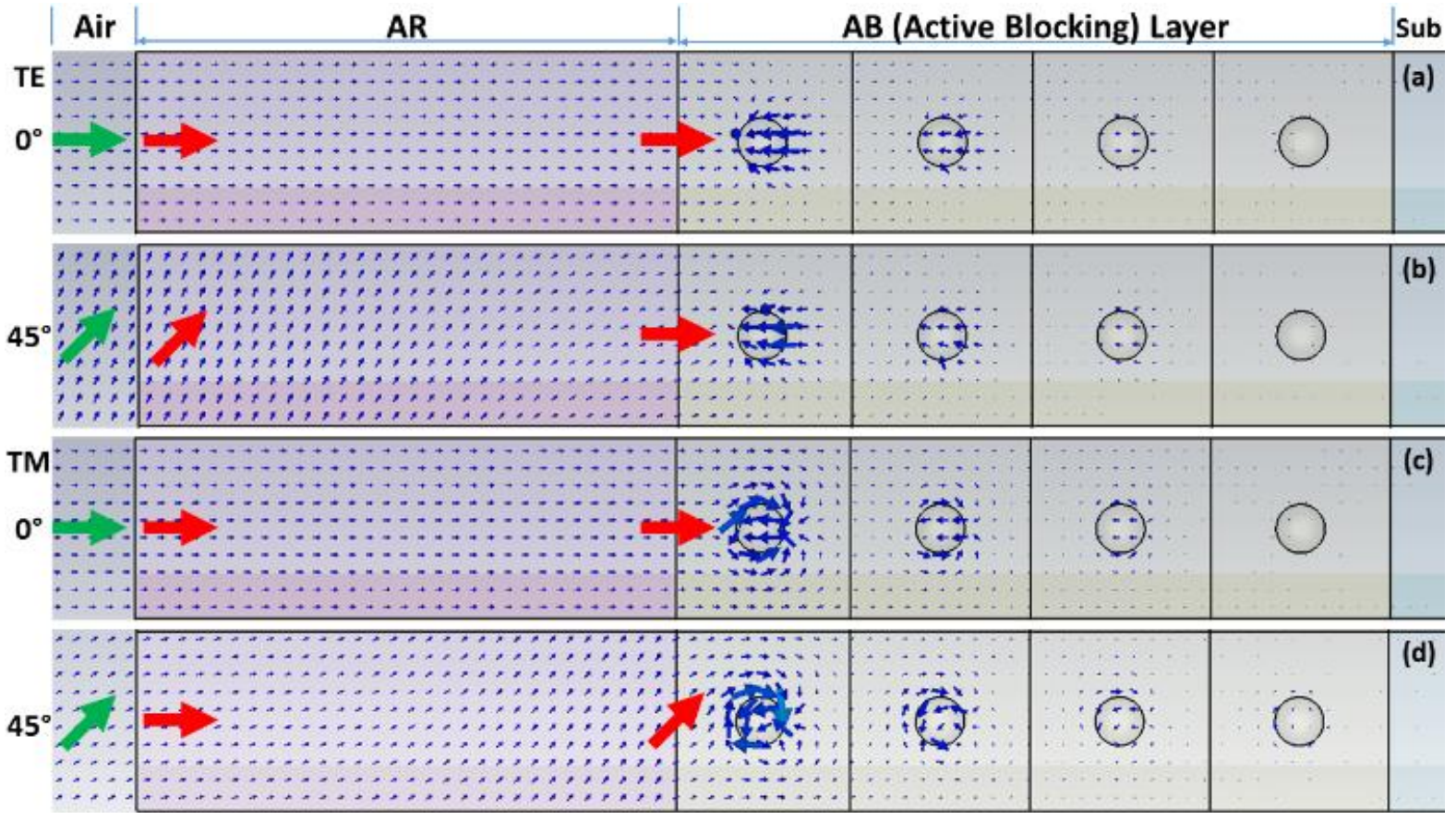

Fig.6. Power flow plots for (a) TE and (b) TM incident beams at 532nm wavelength with varying angles of incident of 0 and 45 degrees.

\section{Discussion}

Manufacturing sub $10 \mathrm{~nm}$ structures on a complex three-dimensional plane presents several major challenges [45], especially when dealing with large area fabrication. Overcoming the diffraction limit that is experienced with conventional photolithography methods is now made possible with the introduction of block co-polymers (BCP), which have gained significant attraction as a nanoscale selfassembling material [46]. The unique ability of BCPs stems from the tendency to assemble discrete ordered morphologies at equilibrium over a large area [47]. Utilising the ability of the BCP to achieve spot feature dimensions of sub $10 \mathrm{~nm}$ [48-50] with a continuously uniform natural period separation could be a progressive move in the fabrication of the described laser protection design. The fabrication process can be enabled with material systems that include PS-b-PMMA, which can exhibit a high-order natural spacing length of $10 \mathrm{~nm}$ to $200 \mathrm{~nm}$ depending on the annealing process [51]. Two-dimensional periodic and symmetric metasurfaces has previously been demonstrated and reported by Ju Young Kim et al. in 2016, where it was successfully demonstrated that a large area assembly of structured plasmonic materials using block copolymer self-assembly method was possible [52]. Moving towards a three-dimensional array can be achieved with multi-layer thin film BCP's [53,54], and would empower the next generation of complex optical metamaterials for various applications. Another possible approach is to use latest dielectric superlens technology with extremely high spatial resolution $(15 \mathrm{~nm})$ coupled with femtosecond laser for direct parallel writing of proposed device in transparent media $[55,56]$.

\section{Conclusion}

Our work explores the advancement of the next generation of laser protection devices and offers a design that is able to block a $532 \mathrm{~nm}$ laser, with an optical density of 1.88+. An additional function to this design is its ability to support multiple polarising laser blocking. The filter is designed using a metamaterial concept, where the active band rejection component is of repeated structures much smaller than the wavelength of interest. The considerable advantage to this filter design is the ability to rejects the lasing wavelength at all angles of incident whilst remaining shift-free, unlike the 
traditional optical thin-film laser protection coatings. Higher optical density can be achieved at the expense of a wider broadband to block pulsed and higher power CW lasers. The transmission of the design from a human's visual perception is $~ 61 \%$, with an overall filter spectral transmission of $78 \%$. This research offers a promising avenue for future high-performance laser protection systems that could be used in a wide variety of industries.

\section{Acknowledgments}

The authors acknowledge the financial support provided by Knowledge Economy Skills Scholarship (KESS2, Ref: BUK289), Wales, United Kingdom, and from Sêr Cymru National Research Network in Advanced Engineering and Materials (NRNF66 and NRN113), Wales, UK.

\section{References}

[1] P. Murphy, "Laser Pointer Safety - Latest aviation statistics", Laserpointersafety.com, 2017. [Online]. Available: http://www.laserpointersafety.com/latest-stats.html. [Accessed: 18- Aug- 2017].

[2] "Laser Incidents reported to the UK CAA 2016," Laser incidents | UK Civil Aviation Authority. [Online]. Available: https://www.caa.co.uk/Data-and-analysis/Safety-and-security/Datasets/Laser-incidents/. [Accessed: 02- Aug- 2017].

[3] V. Nakagawara, R. Montgomery and K. Wood, "Laser Illumination of Flight Crewmembers by Altitude and Chronology of Occurrence", Aviation, Space, and Environmental Medicine, vol. 82, no. 11, pp. 1055-1060, 2011.

[4] J. Coelho, J. Freitas and C. Williamson, "Optical eye simulator for laser dazzle events", Applied Optics, vol. 55, no. 9, p. 2240, 2016.

[5] C. Williamson and L. McLin, "Laser eye dazzle safety framework", in International Laser Safety Conference (ILSC), Atlanta, USA, 2017, p. Paper \#303.

[6] D. Titteton, Military Laser Technology and Systems, 1st ed. Artech House, 2015, pp. 496-506.

[7] M. Chen, C. Li, M. Xu, W. Wang, S. Ma and Y. Xia, "Eye-protection glasses against YAG laser injury based on the band gap reflection of one-dimensional photonic crystal", Optics \& Laser Technology, vol. 39, no. 1, pp. 214-218, 2007.

[8] A. Richter, "Characteristic features of laser-produced plasmas for thin film deposition", Thin Solid Films, vol. 188, no. 2, pp. 275-292, 1990.

[9] A. Thetford, "Thin-film optical filters", Optics Technology, vol. 2, no. 3, 1970.

[10] H. Nalwa, Handbook of thin film materials. San Diego: Academic Press, 2002.

[11] H. Macleod, Thin-film optical filters, 4th ed. CRC Press, 2010, pp. 299-401.

[12] F. Shan, B. Kim, G. Liu, Z. Liu, J. Sohn, W. Lee, B. Shin and Y. Yu, "Blueshift of near band edge emission in Mg doped ZnO thin films and aging", Journal of Applied Physics, vol. 95, no. 9, pp. 4772-4776, 2004.

[13] B. Bovard, "Rugate filter theory: an overview", Applied Optics, vol. 32, no. 28, p. 5427, 1993.

[14] M. Hedayati, F. Faupel and M. Elbahri, "Review of Plasmonic Nanocomposite Metamaterial Absorber", Materials, vol. 7, no. 2, pp. 1221-1248, 2014.

[15] M. Gil, J. Bonache and F. Martín, "Metamaterial filters: A review", Metamaterials, vol. 2, no. 4, pp. 186-197, 2008.

[16] J. Pendry, A. Holden, D. Robbins and W. Stewart, "Magnetism from conductors and enhanced nonlinear phenomena", IEEE Transactions on Microwave Theory and Techniques, vol. 47, no. 11, pp. 2075-2084, 1999.

[17] F. Aznar, M. Gil, J. Bonache and F. Martín, "On the effects of resonator's electrical size on bandwidth in resonant-type metamaterial transmission lines", Microwave and Optical Technology Letters, vol. 52, no. 7, pp. 1526-1530, 2010.

[18] M. Gil, J. Bonache, I. Gil, J. García-García and F. Martín, "On the transmission properties of left-handed microstrip lines implemented by complementary split rings resonators", International Journal of Numerical Modelling: Electronic Networks, Devices and Fields, vol. 19, no. 2, pp. 87-103, 2006.

[19] M. Gil, I. Gil, J. Bonache, J. García-García and F. Martín, "Metamaterial transmission lines with extreme impedance values", Microwave and Optical Technology Letters, vol. 48, no. 12, pp. 2499-2506, 2006.

[20] M. Antoniades and G. Eleftheriades, "A broadband series power divider using zero-degree metamaterial phase-shifting lines", IEEE Microwave and Wireless Components Letters, vol. 15, no. 11, pp. 808-810, 2005.

[21] J. Yang, C. Sauvan, H. Liu and P. Lalanne, "Theory of Fishnet Negative-Index Optical Metamaterials", Physical Review Letters, vol. 107, no. 4, 2011.

[22] J. Valentine, S. Zhang, T. Zentgraf, E. Ulin-Avila, D. Genov, G. Bartal and X. Zhang, "Three-dimensional optical metamaterial with a negative refractive index", Nature, vol. 455, no. 7211, pp. 376-379, 2008.

[23] N. Zhang and Z. Deng, "Method to design microwave band-stop filter based on CPW", Electronics Letters, vol. 47, no. 7, p. 450, 2011.

[24] Z. Bai, Q. Zhang, Y. Ju, G. Tao, X. Jiang, N. Kang, C. Liu and L. Zhang, "Flexible metamaterial narrow-band-pass filter based on magnetic resonance coupling between ultra-thin bilayer frequency selective surfaces", Journal of Physics D: Applied Physics, vol. 49, no. 6, p. 065002, 2015. 
[25] O. Paul, R. Beigang and M. Rahm, "Highly selective terahertz bandpass filters based on trapped mode excitation", Optics Express, vol. 17, no. 21, p. 18590, 2009.

[26] A. Horestani, W. Withayachumnankul, A. Chahadih, A. Ghaddar, M. Zehar, D. Abbott, C. Fumeaux and T. Akalin, "Metamaterial-Inspired Bandpass Filters for Terahertz Surface Waves on Goubau Lines", IEEE Transactions on Terahertz Science and Technology, vol. 3, no. 6, pp. 851-858, 2013.

[27] A. Sakurai, B. Zhao and Z.M. Zhang, "Prediction of the Resonance Condition of Metamaterial Emitters and Absorbers using LC Circuit Model”, in International Heat Transfer Conference, Kyoto, Japan, 2014, IHTC15-9012.

[28] G. Dayal and S. Ramakrishna, "Broadband infrared metamaterial absorber with visible transparency using ITO as ground plane", Optics Express, vol. 22, no. 12, p. 15104, 2014.

[29] S. Jahani and Z. Jacob, "All-dielectric metamaterials", Nature Nanotechnology, vol. 11, no. 1, pp. 23-26, 2016.

[30] X. Li, L. Yang, C. Hu, X. Luo and M. Hong, "Tunable bandwidth of band-stop filter by metamaterial cell coupling in optical frequency", Optics Express, vol. 19, no. 6, p. 5283, 2011.

[31] N. Liu, M. Mesch, T. Weiss, M. Hentschel and H. Giessen, "Infrared Perfect Absorber and Its Application as Plasmonic Sensor", Nano Letters, vol. 10, no. 7, pp. 2342-2348, 2010.

[32] S.Zhang, W. Fan, N. Panoiu, K. Malloy, R. Osgood and S. Brueck, "Optical negative-index bulk metamaterials consisting of 2D perforated metal-dielectric stacks", Optical Express, vol. 14, no. 15, p. 6778, 2006.

[33] N. Liu, H. Guo, L. Fu, S. Kaiser, H. Schweizer and H. Giessen, "Three-dimensional photonic metamaterials at optical frequencies", Nature Materials, vol. 7, no. 1, pp. 31-37, 2007.

[34] W. Cai and V. Shalaev, Optical metamaterials: Fundamentals and Applications. Springer, 2010, pp. 25-36.

[35] D. Gall, "Electron mean free path in elemental metals", Journal of Applied Physics, vol. 119, no. 8, p. $085101,2016$.

[36] I. Moreno, J. Araiza and M. Avendano-Alejo, "Thin-film spatial filters", Optical Letters, vol. 30, no. 8, p.914, 2005.

[37] H. Macleod, Thin-film optical filters, 4th ed. CRC Press, 2010, pp. 105-184.

[38] "CST STUDIO SUITE - Market Leading Electromagnetic Simulation Software", Cst.com, 2017. [Online]. Available: https://www.cst.com/products/cst2. [Accessed: 08- Oct- 2017].

[39] Y. Jiang, S. Pillai and M. Green, "Re-evaluation of literature values of silver optical constants", Optics Express, vol. 23, no. 3, p. 2133, 2015.

[40] M. Dodge, "Refractive properties of magnesium fluoride", Applied Optics, vol. 23, no. 12, p. 1980, 1984.

[41] M. Querry, "Optical Constants of Minerals and Other Materials from the Millimeter to the Ultraviolet", (Dover, NJ, U.S. Army Armament, Munitions Chemical Research), 1987.

[42] U. Kreibig, "Kramers Kronig analysis of the optical properties of small silver particles", Zeitschrift Physik, vol. 234, no. 4, pp. 307-318, 1970.

[43] H. Mahlein, "Generalized Brewster-angle conditions for quarter-wave multilayers at non-normal incidence*", Journal of the Optical Society of America, vol. 64, no. 5, p. 647, 1974.

[44] A. Maradudin, Structured Surfaces as Optical Metamaterials. Cambridge: Cambridge University Press, 2011, pp. 58-93.

[45] X. Zhang, C. Sun and N. Fang, "Manufacturing at Nanoscale: Top-Down, Bottom-up and System Engineering", Journal of Nanoparticle Research, vol. 6, no. 1, pp. 125-130, 2004.

[46] M. Park, C. Harrison, P. Chaikin, R. Register and D. Adamson, "Block Copolymer Lithography: Periodic Arrays of 1011 Holes in 1 Square Centimeter", Science, vol. 276, no. 5317, pp. 1401-1404, 1997.

[47] F. Bates and G. Fredrickson, "Block Copolymer Thermodynamics: Theory and Experiment", Annual Review of Physical Chemistry, vol. 41, no. 1, pp. 525-557, 1990.

[48] M. Kim and G. Yi, "Nanostructured Colloidal Particles by Confined Self-Assembly of Block Copolymers in Evaporative Droplets", Frontiers in Materials, vol. 2, 2015.

[49] A. Horechyy, B. Nandan, N. Zafeiropoulos, P. Formanek, U. Oerel, N. Bigall, A. Eychmuller and M. Stamm, "A Step-Wise Approach for Dual Nanoparticle Patterning via Block Copolymer Self-Assembly", Advanced Functional Materials, vol. 23, no. 4, pp. 483-490, 2012.

[50] J. Chai, F. Huo, Z. Zheng, L. Giam, W. Shim and C. Mirkin, "Scanning probe block copolymer lithography", Proceedings of the National Academy of Science, vol. 107, no. 47, pp. 20202-20206, 2010.

[51] F. Ferrarese Lupi, T. Giammaria, F. Volpe, F. Lotto, G. Seguini, B. Pivac, M. Laus and M. Perego, “High Aspect Ratio PS-bPMMA Block Copolymer Mask for Lithographic Applications", ACS Applied Materials \& Interfaces, vol. 6, no.23, pp. 2138921396, 2014.

[52] J. Kim, H. Kim, B. Kim, T. Chang, J. Lim, H. Jin, J. Mun, Y. Choi, K. Chung, J. Shin, S. Fan and S. Kim, “Highly tunable refractive index visible-light metasurface from block copolymer self-assembly", Nature Communications, vol. 7, p. 12911, 2016.

[53] A. Tavakkoli, S. Nicaise, K. Gadelrab, A. Alexander-Katz, C. Ross and K. Berggren, "Multilayer block copolymer meshes by orthogonal self-assembly", Nature Communications, vol. 7, p. 10518, 2016.

[54] B. Kuila, P. Formanek and M. Stamm, "Multilayer polymer thin films for fabrication of ordered multifunctional polymer nanocomposites", Nanoscale, vol. 5, no. 22, p. 10849, 2013. 
[55] W. Fan, B. Yan, Z. Wang and L. Wu, "Three-dimensional all-dielectric metamaterial solid immersion lens for subwavelength imaging at visible frequencies", Science Advances, vol. 2, no. 8, 2016.

[56] Z. Wang, W. Guo, A. Pena, D. Whitehead, B. Luk'yanchuk, L. Li, Z. Liu, Y. Zhou and M. Hong, "Laser micro/nano fabrication in glass with tunable-focus particle lens array", Optics Express, vol. 16, no. 24, p. 19706, 2008. 